\title{
Nasopharyngeal Carriage of Staphylococcus aureus among Horses and Horse Handlers in Kano Metropolis, Nigeria
}

Sanda, M. $I^{* 1}$ and Idris, A. $M^{2}$.

${ }^{1}$ Department of Animal Sciences, Faculty of Agricultural Sciences, Bayero University, Kano, Nigeria ${ }^{2}$ Department of Medical Microbiology and Parasitology, Faculty of Clinical Sciences, College of Health Science, Bayero University, Kano, Nigeria

Abstract
Staphylococcal species are important opportunistic bacterial pathogens that can asymptomatically
colonize both human and animals bodies. The presence of nasopharyngeal carriage Staphylococci
has been an increased risk factor of acquiring an infection with this pathogen. The present study
aimed at determining the nasopharyngeal carriage of Staphylococcal aureus in horses and horse
handlers in Kano metropolis, Nigeria. One hundred and sixty (160) non-duplicated nasopharyngeal
samples were collected using sterile swab stick from each horse and 100 from consented horse
handlers. All the samples were analyzed using a standard bacteriological procedure. Antibiotics
susceptibility testing to eight (8) most commonly used antibiotics was carried out using a modified
Kirby Bauer method. One hundred and forty-two (88.8\%) staphylococcal isolates were recovered
from horses and 84 (84.0\%) from horse handlers. Among all the isolated staphylococci high
sensitivity was observed in response to Gentamycin and Ciprofloxacin antibiotics, while
Tetracycline was found to have the least activity. The report of this study showed a high
prevalence of Staphylococci among horses and horse handlers. Moreover, it confirmed the
tendency of Staphylococcal isolates cross-transmission between horses and handlers in the study
site. This study indicated the importance of increase in handler's awareness of possible risk
factors of staphylococcal colonization that can lead to invasive infection.
Keywords: Antibiotics, Horses, Horse Handlers, Nasopharyngeal, Staphylococci

\section{INTRODUCTION}

Staphylococcus aureus is an important opportunistic bacterial pathogen that can asymptomatically colonize in both human and diverse animals (Saei and Safari, 2019). Among the human, S. aureus is a common colonizer of the skin, nasal cavity, and other mucosal membranes (Wertheim et al., 2005; Agabou et al., 2017). In animal domains, S. aureus has commonly inhabited the nostrils, nares, mouth, and perineum (Iverson et al., 2015; Agabou et al., 2017).

Nasal carriage Staphylococci play a major role in the pathogenicity of the Staphylococcal infections, in hospitalized patients especially among those who undergo delicate procedures such as surgery, dialysis, and patients in an intensive care unit (ICU) that have higher infection risks in persistent carriers (Sakr et al., 2018). A study done by Van Belkum et al. (2009) reported that carriers of staphylococci are between 3 - 6 times more likely to develop a chronic staphylococcal infection than non-carriers (Van Belkum et al., 2009; Malley et al., 2015). Moreover, about $80.0 \%$ of the invasive staphylococcal infections have resulted from colonizer of the host normal flora (O'Malley et al., 2015).

Staphylococcus aureus becomes one of the major public health problems worldwide, due to its high morbidity and mortality rates and its ability to resist different classes of antibiotics which were initially recommended for their treatment (Gaddafi et al., 2020). Antibiotics resistance in staphylococci has developed through spontaneous mutations, enzymatic inactivation of the drugs, and target site alteration which reduces binding affinity (Pantosti et al., 2007). There is increasing evidence that shifts the spread of resistance staphylococcal infection from hospitalassociated infection to a community-associated infection as a result of increased contact between humans and productive livestock, pet animals, and horses inside and outside the hospital settings (Cuny et al., 2006). Klous et al. (2016) in their study reported that zoonotic infections account for an estimated $60.0 \%$ of all human infections (Klous et al., 2016).

In Nigeria, there has been increased contact between human beings and horses, for recreational, traditional, sports, and breeding purposes over the years (Abdulkadir, 2014). 
Prevention of the spread of staphylococcal infection among the people requires accurate evaluation of the nature and type of resistance in both the horses and their handlers and by gaining insight into the transmission routes. Since horses can serve as sources of infection and/or re-infection of humans, with physical contact between humans and horses being unavoidable, adequate and routine assessments of staphylococcal colonization in both handlers and their horses are required for efficient control of the infection. The present study aimed at determining the nasopharyngeal carriage of Staphylococcalaureus in horses and horse handlers in Kano metropolis, Nigeria.

\section{MATERIALS AND METHODS}

\section{Study Area}

The study area was carried out at Kano metropolis, Nigeria. The state is located in Northwestern Nigeria on latitude 8o 30' $\mathrm{E}$ and longitude $11030^{\prime} \mathrm{N}, 402 \mathrm{~m}$ above sea level (Kano Google satellite map, 2016). It has distinct wet and dry seasons within the guinea and part of the Sahel savannah zones of Nigeria. Kano is the commercial center of the horse business in Nigeria. Most of the imported breeds from Argentina, South Africa, Sudan, Cameroon, and Niger are domiciled in Kano for distribution to other states. Kano is the home of Durbar, leisure riding, polo, and racing and all horse activities take place throughout the year. It is near two neighboring international horse markets of Mai'Adua and Maigatari from where different breeds of horses usually find their way to the Kano metropolis (NBS, 2018).

\section{Study Design}

This was a cross-sectional study and a convenience sampling of clinically normal horses selected from the study area. The horses selected were in three categories; performance horses (polo and racing horses), traditional horses that are kept locally for recreation and cultural activities. Animals were sampled only after owners' consent was given. All samples were collected by a trained technician.

Microbiological Analysis

Data Collection

Self-administered structured questionnaires were given to each participant after consent.

\section{Sample Collection}

A total of 160 non duplicated nasopharyngeal samples were collected using sterile swab sticks from each horse and 100 from a human participant. A moistened cotton-tipped swab stick was inserted approximately $2 \mathrm{~cm}$ for humans and $10 \mathrm{~cm}$ for horses in one nasal passage, rotated for about 10 seconds and withdrawn with the swab in contact with the nasal mucosa. The swab was promptly placed in liquid Stuart's medium. The swab was kept at $40 \mathrm{C}$ in a Coleman box and transported to the Microbiology Laboratory and processed according to standard microbiological procedures (Cheesbrough, 2010).

Media Preparation

All the media were prepared according to the manufacturer's instructions.

\section{Gram Staining}

Gram staining of the isolated colonies was carried out to identify the Gram reaction of the isolates as described by (Cheesbrough, 2010).

\section{Biochemical Tests}

Suspected Staphylococcal isolates were identified and confirmed using standard bacteriological procedures.

Catalase Test

This test is used to differentiate those bacteria that produce Catalase enzymes (e.g Staphylococci) from non-Catalase-producing bacteria (eg. Streptococci) (Cheesbrough, 2010).

\section{Coagulase Test}

This test is used to differentiate members of the genus staphylococcus such as Staphylococcus aureus that produce coagulase enzymes and other non-coagulase negative species (Cheesbrough, 2010).

\section{Hemolysis Activities}

Hemolysis activity of the member of the genus staphylococci was detected using blood agar. A blood agar base (Oxoid, UK) was prepared according to the manufacture's guidelines. About $20 \mathrm{ml}$ volume of the prepared blood agar was poured into Petri dishes and dried off surface moisture. A colony of the Staphylococci under test was touched with an inoculating loop, streak on the plate, and incubated at $37^{\circ} \mathrm{C}$ for 24 hours in $-5 \% \mathrm{CO}$. The plate was checked for the presence of hemolysis activity (Cheesbrough, 2010).

\section{Mannitol Fermentation}

Mannitol salt agar was prepared according to the manufactures direction. The isolate was subcultured on the medium incubated for 24 hours at $37^{\circ} \mathrm{C}$. The plate was observed for mannitol fermentation by the isolates after the incubation (Cheesbrough, 2010).

Preparation of McFarland Turbidity Standard

Preparation of 0.5 McFarland standard (turbidity standard) Sulfuric sulfate $(1 \% \mathrm{v} / \mathrm{v})$ standard suspension was used as turbidity standard which was prepared following the procedure explained by Cheesbrough (2010).

Antibiotic sensitivity testing

Antimicrobial susceptibility testing against 8 most common used antibiotics was done using the modified Kirby-Bauer disk diffusion method on Mueller Hinton agar based on the Clinical and Laboratory Standards Institute (CLSI, 2019). 
The antibiotics (Oxoid, UK) used include

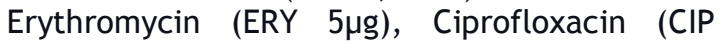

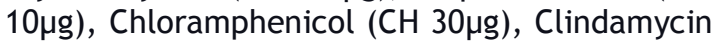

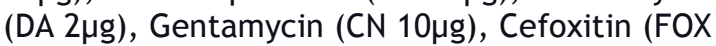

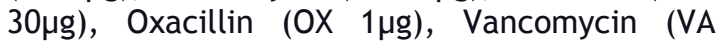

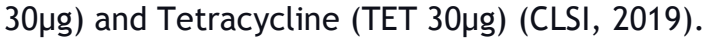
Data Analysis

The data generated in this study were analyzed using the Statistical Package for Social Sciences (SPSS) for windows version 20.0 used for statistical analysis and data interpretation. The values were expressed as means and percentages. Comparison of variables was done using Chi-square test and values with $\mathrm{p} \leq 0.05$ were considered significant.

\section{RESULTS}

A total of 160 horses and 100 horse handlers were enrolled in the study, the higher number of the participants is $80(50.0 \%)$ horses and 47 (52.2\%) from the category traditional horse activities (Durba). Institutional horses and their handlers have the least number of participants $2(1.3 \%)$ and $3(3.3 \%)$ respectively (Table 1$)$. Among the samples collected from horses, 142 $(88.8 \%)$ yield positive cultures and 84 (84.0\%) were culture positive from horse handlers. This gives the overall nasopharyngeal colonization of 88.8\% among horses and $84.0 \%$ among humans handling the horses in the study site (Table 2 ).
All the $142(100.0 \%)$ isolates obtained from horses were Gram-positive cocci in pair and cluster and Catalase positive. Out of the 142 isolates, $125(88.0 \%)$ were positive for slide and tube coagulase test, 47 (33.1\%) showed betahemolytic, and 129 (90.9\%) ferment mannitol when growing on mannitol salt agar. From 84 positive cultures found in horse handlers, 84 $(100.0 \%)$ were both Gram-positive cocci in pair and cluster and Catalase positive. Thirty-three (39.3\%) coagulase-positive, 20 (23.8\%) showed beta-hemolytic, and 33 (39.3\%) ferment mannitol (Table 3).

Table 4 shows the rate of occurrence of Staphylococcal nasopharyngeal carriage with regards to horse activity. The highest occurrence was obtained among institute horses 2 (100.0\%) and horse handlers 3 (100.0\%) and also $20(100.0 \%)$ racing horse handlers, followed by polo horses 36 (97.3\%) in horses and $43(91.5 \%)$ horse handlers in traditional horse activities. Statistically in all the two groups, no significant relationship was obtained at a 95\% confidence interval as $p$-value $>0.05$. Antibiotics susceptibility profile of the isolated staphylococci obtained from both horses and horse handlers using conventional antibiotics shows high activity to Gentamycin $(10 \mu \mathrm{g})$ and Ciprofloxacin $(10 \mu \mathrm{g})$ and least activity was observed in Tetracycline $(30 \mu \mathrm{g})$ (Table 5).

Table 1: Classification of Horses and Horse handlers according to Category of Horse Activities

\begin{tabular}{lll}
\hline Horse Activity & Horses $(\%)$ & Horse Handlers (\%) \\
\hline Institution & $2(1.3)$ & $3(3.3)$ \\
Polo & $37(23.1)$ & $20(22.2)$ \\
Racing & $41(25.6)$ & $20(22.2)$ \\
Traditional & $80(50.0)$ & $47(52.2)$ \\
Total & $160(100.0)$ & $90(100.0)$ \\
\hline
\end{tabular}

Table 2: Prevalence of Staphylococci Colonizer in Horses and Horse Handlers in Kano Metropolis

\begin{tabular}{llll}
\hline & No. Examined & No. Positive (\%) & No. Negative (\%) \\
\hline Horse & 160 & $142(88.8)$ & $18(11.3)$ \\
Horse Handlers & 100 & $84(84.0)$ & $16(16.0)$ \\
\hline
\end{tabular}

Table 3: Phenotypic Characteristics of the Staphylococcal Isolates

\begin{tabular}{lll}
\hline Tests & Horse $(\mathrm{n}=142)$ & Horse Handlers $(\mathrm{n}=84)$ \\
\cline { 2 - 3 } & No. Positive $(\%)$ & No. Positive $(\%)$ \\
\hline Gram's Reaction & $142(100.0)$ & $84(100.0)$ \\
Catalase Test & $142(100.0)$ & $84(100.0)$ \\
Coagulase Test & $125(88.0)$ & $33(39.3)$ \\
Haemolysis Activity & $47(33.1)$ & $20(23.8)$ \\
Mannitol Fermentation & $129(90.9)$ & $33(39.3)$ \\
\hline
\end{tabular}


UJMR, Volume 6 Number 1, June, 2021, pp 195 - $200 \quad$ ISSN: 2616 - 0668

Table 4: Distribution of Staphylococcal Nasopharyngeal Carriage among Horses and Horse Handlers

\begin{tabular}{lllll}
\hline Horse Activity & Horse & \multicolumn{3}{c}{ Horse Handlers } \\
\hline Horse Activity & No. Examined & Positive $(\%)$ & No. Examined & Positive (\%) \\
Institution & 2 & $2(100.0)$ & 3 & $3(100.0)$ \\
Polo & 37 & $36(97.3)$ & 20 & $18(90.0)$ \\
Racing & 41 & $37(90.2)$ & 20 & $20(100.0)$ \\
Traditional & 80 & $67(83.8)$ & 47 & $43(91.5)$ \\
Total & 160 & $142(88.8)$ & 90 & $84(93.3)$ \\
$P$-value & ${ }^{*} 0.961$ & & ${ }^{* *} 0.978$ & \\
${ }^{*} \times 2=0.297 \mathrm{df}=3$ P-value $=0.961$ & & & \\
${ }^{* *} \times 2=0.200 \mathrm{df}=3$ P-value $=0.978$ & & &
\end{tabular}

Table 5: Antibiotics Susceptibility Pattern of Staphylococcal Isolates

\begin{tabular}{lllllll}
\hline Antibiotics $(\mu \mathrm{g})$ & \multicolumn{5}{l}{ Horse N $=142(\%)$} & \multicolumn{4}{l}{ Horse Handlers N $=84(\%)$} \\
\cline { 2 - 7 } & $\mathrm{S}$ & $\mathrm{I}$ & $\mathrm{R}$ & $\mathrm{S}$ & $\mathrm{I}$ & $\mathrm{R}$ \\
\hline ERY $(5)$ & $116(81.7)$ & $9(6.3)$ & $17(12.0)$ & $71(84.5)$ & $7(8.3)$ & $6(7.1)$ \\
GEN $(10)$ & $141(99.3)$ & $1(0.7)$ & $0(0.0)$ & $81(96.4)$ & $0(0.0)$ & $3(3.6)$ \\
CH $(30)$ & $123(86.6)$ & $10(7.0)$ & $9(6.3)$ & $77(91.7)$ & $1(1.2)$ & $6(7.1)$ \\
TET $(30)$ & $51(35.9)$ & $18(12.7)$ & $73(51.4)$ & $50(59.5)$ & $8(9.5)$ & $26(31.0)$ \\
CIP (10) & $138(97.2)$ & $2(1.4)$ & $2(1.4)$ & $82(97.6)$ & $0(0.0)$ & $2(2.4)$ \\
DA (2) & $102(71.8)$ & $38(26.8)$ & $2(1.4)$ & $76(90.5)$ & $5(6.0)$ & $3(3.6)$ \\
VA (30) & $102(71.8)$ & $28(19.7)$ & $12(8.5)$ & $68(81.0)$ & $10(11.9)$ & $6(7.1)$ \\
FOX (30) & $133(93.7)$ & $0(0.0)$ & $9(6.3)$ & $66(78.6)$ & $0(0.0)$ & $18(21.4)$ \\
\hline
\end{tabular}

Key: $\mathrm{S}=$ Sensitivity, I = Intermediate, $\mathrm{R}=$ Resistance, ERY = Erythromycin, GEN = Gentamycin, $\mathrm{CH}=$ Chloramphenicol, TET = Tetracycline, $\mathrm{CIP}=$ Ciprofloxacin, $\mathrm{DA}=$ Clindamycin, $\mathrm{VA}=$ Vancomycin, FOX $=$ Cefoxitin, $\mathrm{OX}=$ Oxacillin

\section{DISCUSSION}

In this study, the overall prevalence rate of nasopharyngeal carriage staphylococci among horses was $88.8 \%$. The prevalence rate reported in this study was much higher than the $20.3 \%$ recorded by Saei and Safari (2019) in Iran, $15.2 \%$ by Agbou et al. (2017) in Algeria, $13.5 \%$ by Islam et al. (2017) in Denmark, 15.4\% recorded by Abdulkadir (2014) in Zaria, Nigeria and $50.0 \%$ by Zunita et al. (2008) in Malaysia. These differences may be due to environmental factors, animal husbandry practices and hygienic status. The outcome of the present study indicated that horses in this study area can serve as a reservoir of staphylococcus species that can lead to zoonotic transmission to the horse handlers.Similar studies by Ansari et al. (2017); Selva et al. (2015) reported that nasopharyngeal colonization by $S$. aureus serves as an important source of staphylococci infection in humans as well as an increased risk factor for developing an invasive infection. Wertheim et al. (2005) earlier reported that nasopharyngeal carriage of staphylococci has been recognized as an increased risk factor of acquiring an infection with this pathogen.

The study findings revealed that he overall prevalence rate of nasopharyngeal carriage of staphylococci among horse handlers was $84.0 \%$, and was found to be higher than the $10.8 \%$ carriage rate among horse handlersin a similar study in Zaria, Kaduna State, by Abdulkadir (2014). It is equally higher than the report of another study in Denmark, which recorded the prevalence rate of $50.0 \%$ among humans handling the horses (Islam et al., 2017).

In this study, the antibiotics susceptibility pattern of staphylococci isolates obtained from horses showed that $99.3 \%$ were sensitive to Gentamycin while $97.2 \%$ and $93.7 \%$ were susceptible to Ciprofloxacin and Cefoxitin, respectively. The isolates were less sensitive to Tetracycline (35.9\%). This result is in agreement with the finding of Agabou et al. (2017) that recorded high sensitivity of Gentamycin (100.0\%) and Ciprofloxacin $(100.0 \%)$, and that of Abdulkadir (2014) that recorded the sensitivity of $93.8 \%$ to Gentamycin and Ciprofloxacin. This study further agrees with the work done by Adegoke and Okoh (2014) that recorded the high resistant of the isolates to Tetracycline (83.3\%).

The staphylococcal isolates obtained from horse handlers showed more susceptibility to Ciprofloxacin $(97.6 \%)$ followed by Gentamycin (96.4\%). They agreed with the study reported by Agabou et al. (2017) and Abdulkadir (2014) that recorded high sensitivity of $100.0 \%$ to Ciprofloxacin and Gentamycin. 


\section{CONCLUSION AND RECOMMENDATIONS}

This study reports a high prevalence of staphylococci carriage among horses and horse handlers. The study identifies the possibility of staphylococcal isolates to cross-transmission between horses and handlers in the study site. The study reported high susceptibility of the

\section{REFERENCES}

Abdulkadir, A. (2014). Methicillin resistant Staphylococcus aureus in horses and horse Handlers in Kaduna and Zaria, Nigeria. An MSc Thesis in the Department of Veterinary Public Health and Preventive Medicine, Ahmadu Bello University, Zaria. Retrieved on $5^{\text {th }}$ January, 2020.

Adegoke, A.A. andOkoh, A.I. (2014). Species diversity and antibiotic resistance properties of Staphylococcus of farm animal origin in Nkonkobe Municipality, South Africa. Folia Microbiology, 59:133-140.

Agabou, A., Ouchenane, Z., NgbaEssebe, C., Khemissi, S., Chehboub, M.T.E., Chehboub, I.B., Sotto, A., DunyachRemy, C. andLavigne, J.P. (2017)Emergence of nasal carriage of ST80 and ST152 PVL+ Staphylococcus aureus isolates from livestock in Algeria. Toxins, 9:303 - 315.

Cheebrough, M.(2010). District Laboratory Practice in Tropical Countries. Cambridge University Press, 2:64-67.

Clinical and Laboratory Standard Institute (CLSI) (2019). Performance Standards for Antimicrobial Susceptibility Testing, Twenty - Seven Informational Supplement.M02-A12, M07-A10, and M11-A8. Clinical and Laboratory Standards Institute 950 West Valley Road, Suite 2500 Wayne, PA 19087 USA, 27:18 - 22.

Cuny, C., Kuemmerle, J., Stanek, C., Willey, B., Strommenger, B. and Witte, W. (2006). Emergence of MRSA Infections In Horses In A Veterinary Hospital: Strain Characterisation And Comparison With Mrsa From Humans. European Centre for Disease Prevention and Control,11(1): 44-47

Gaddafi, M.S., Yakubu,Y., Garba, B., Bello, M.B., Musawa, A.I. and Lawal, H. (2020). Occurrence and antimicrobial resistant patterns of methicillin resistant Staphylococcus aureus (MRSA) among practicing veterinarians in Kebbi state, Nigeria. FoliaVeterinarian, 64(4): 55-62.

UMYU Journal of Microbiology Research staphylococcal isolates to Ciprofloxacin and Gentamycin and high resistance to tetracycline. The study indicated the importance of increasing the handler's awareness of possible risk factors of staphylococcal colonization that can lead to invasive infection.

Islam,M.Z, Espinosa-Gongora, C., Damborg, P., Sieber, R.N.,Munk, R., Husted, L., Moodley, A., Skov, R., Larsen, J. andGuardabassi, L. (2017). Horses in Denmark are a reservoir of diverse clones of methicillin-resistant and susceptible Staphylococcusaureus. Front Microbiology, 8:543

Iverson, S.A., Brazil, A.M., Ferguson, J.M., Nelson, K., Lautenbach, E., Rankin, S.C., Morris, D.O. and Davis, M.F. (2015). Anatomical patterns of colonization of pets with staphylococcal species in homes of people with methicillin-resistant Staphylococcusaureus (MRSA) skin or soft tissue infection (SSTI). Veterinary Microbiology, 176:202208.

Klous, G., Huss, A., Heederik, D.J.J. and Coutinho, R.A. (2016). Humanlivestock contacts and their relationship to transmission of zoonotic pathogens, a systematic review of literature. One Health, 2: 65-76.

National Bureau of Statistics (NBS) (2018). The latest population figures from National Bureau of Statistics you need to see; Business Insider by Pulse; Retrieved on $15^{\text {th }}$ December 2019.

O'Malleya, S.M., Emeleb, F.E., Nwaokoriec, F.O., Idika, N., Umeizudike, A.K., Emeka-Nwabunniae, I., Hanson, B.M., Nair, R., Wardyn, S.E. and Smith, T.C. (2015). Molecular typing of antibiotic-resistant Staphylococcus aureus in Nigeria. Journal of Infection and Public Health, 8:187193.

Pantosti, A., Sanchini, A. and Monaco, M. (2007). Mechanisms of antibiotic resistance in Staphylococcus aureus. Future Microbiology, 2(3):323-334

Saei, H.D. and Safari, E. (2019). Methicillin resistance and clonal diversity of Staphylococcusaureus isolated from nasal samples of healthy horses in Iran. Annals of Microbiology, 69:923931 
Sakr, A., Brégeon, F., Mège, J.L., Rolain, J.M. and Blin, $0 . \quad$ (2018). Staphylococcusaureus Nasal Colonization: An Update on Mechanisms, Epidemiology, Risk Factors, and Subsequent Infections. Front Microbiology, 9:2419

Selva, L., Viana, D. and Corpa, J.M. (2015). Staphylococcus aureus nasal carriage could be a risk for development of clinical infections in rabbits. World Rabbit Sciences, 23:181-184.

Van Belkum, A., Melles, D.C., Nouwen, J., Van Leeuwen, W.B., Van Wamel,W., Vos, M.C., Wertheim, H.F. andVerbrugh, H.A. (2009). Co- evolutionary aspects of human colonization and infection by Staphylococcusaureus. InfectiousGeneticEvolution, 9:32-47. Wertheim, H.F., Melles, D.C., Vos, M.C., Van Leeuwen, W., Van Belkum, A., Verbrugh, H.A. and Nouwen, J.L. (2005). The role of nasal carriage in Staphylococcusaureus infections. Lancet Infectious Disease, 5:751-762.

Zunita, Z., Bashir, A., and Hafizal, A. (2008) Occurrence of multidrug resistant Staphylococcusaureus in horses in Malaysia. Veterinary World, 1:165167. 